\title{
Controlled Magnetic Anisotropy in Single Domain Mn-doped Biosynthesized Nanoparticles
}

\author{
Lourdes Marcano,* Iñaki Orue, Ana García-Prieto, Radu Abrudan, Javier Alonso, \\ Luis Fernández Barquín, Sergio Valencia, Alicia Muela, and M. Luisa Fdez-Gubieda
}

Cite This: J. Phys. Chem. C 2020, 124, 22827-22838

Read Online

ACCESS

Lill Metrics \& More

Article Recommendations

Supporting Information

ABSTRACT: Magnetotactic bacteria Magnetospirillum gryphiswaldense synthesize cubo-octahedral shaped magnetite nanoparticles, called magnetosomes, with a mean diameter of $40 \mathrm{~nm}$. The high quality of the biosynthesized nanoparticles makes them suitable for numerous applications in fields like cancer therapy, among others. The magnetic properties of magnetite magnetosomes can be tailored by doping them with transition metal elements, increasing their potential applications. In this work, we address the effect of $\mathrm{Mn}$ doping on the main properties of magnetosomes by the combination of structural and magnetic characterization techniques. Energy-dispersive X-ray spectroscopy, X-ray absorption near-

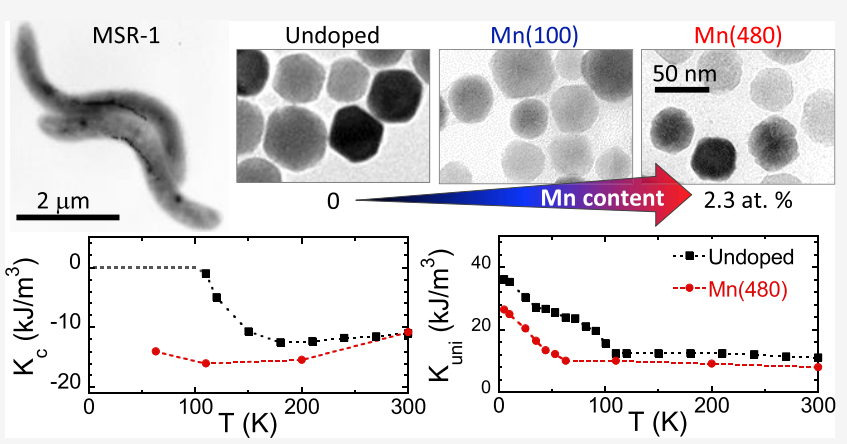
edge structure, and $\mathrm{X}$-ray magnetic circular dichroism results reveal a $\mathrm{Mn}$ dopant percentage of utmost $2.3 \%$, where $\mathrm{Mn}$ cations are incorporated as a combination of $\mathrm{Mn}^{2+}$ and $\mathrm{Mn}^{3+}$, preferably occupying tetrahedral and octahedral sites, respectively. Fe substitution by Mn notably alters the magnetic behavior of the doped magnetosomes. Theoretical modeling of the experimental hysteresis loops taken between 5 and $300 \mathrm{~K}$ with a modified StonerWohlfarth approach highlights the different anisotropy contributions of the doped magnetosomes as a function of temperature. In comparison with the undoped magnetosomes, $\mathrm{Mn}$ incorporation alters the magnetocrystalline anisotropy introducing a negative and larger cubic anisotropy down to the Verwey transition, which appears shifted to lower temperature values as a consequence of Mn doping. On the other hand, Mn-doped magnetosomes show a decrease in the uniaxial anisotropy in the whole temperature range, most likely associated with a morphological modification of the Mn-doped magnetosomes.

\section{INTRODUCTION}

Magnetotactic bacteria are a group of aquatic microorganisms widespread in freshwater and marine environments that can passively align in the presence of magnetic fields while they actively swim. This fascinating behavior is due to the presence of intracellular membrane-enclosed single domain magnetic nanoparticles, called magnetosomes, arranged in chains. ${ }^{1-4}$ Magnetosomes present a species-specific morphology and size, and a precise mineral composition, in most cases magnetite. The biological precision exhibited in the synthesis process bestows exceptional properties on magnetosomes, which attracts a lot of scientific attention, being excellent candidates for biomedical applications. ${ }^{5-9}$ In addition, recent studies have addressed the possibility of exploiting the whole magnetotactic bacteria as microrobots for cancer treatment. ${ }^{10-12}$ These microorganisms provide a self-propelling mechanism secured by their flagella and the advantage to be manipulated and guided, thanks to the presence of the magnetosome chain. Furthermore, the biological structure of the magnetosome chain of magnetotactic bacteria enhances the hyperthermia efficiency compared with isolated magnetosomes and their synthetic counterparts. ${ }^{13}$
Aiming to expand the range of applications of magnetosomes and magnetotactic bacteria, the past decade has witnessed a huge growth in the development of different strategies to tailor magnetosome properties, which are otherwise set by the genetic control of the bacteria. The substitution of iron cations by transition metal dopants in magnetite serves as a proven method to change the magnetic properties of the magnetic nanoparticles. ${ }^{14}$ Under this premise, Staniland et al. ${ }^{15}$ pioneered the in vivo Co doping of magnetosomes under laboratory conditions by simply adding certain amounts of this element into the culture medium. ${ }^{15}$ Since then, several groups have been able to dope magnetosomes with different transition metals such as $\mathrm{Co}, \mathrm{Mn}$, and $\mathrm{Cu}^{16-22}$

Received: July 31,2020

Revised: September 12, 2020

Published: September 15, 2020 
To this respect, doping the magnetosomes with $\mathrm{Mn}$ is especially attractive due to the higher magnetization with respect to magnetite, lower coercivity, and proven biocompatibility evidenced in synthetic Mn-substituted magnetite $\left(\mathrm{Mn}_{x} \mathrm{Fe}_{3-x} \mathrm{O}_{4}\right)$ nanoparticles. ${ }^{23-25}$ There are several reports in the literature addressing the incorporation of $\mathrm{Mn}$ into the inner structure of magnetosomes synthesized by different bacterial strains. Keim et al. first evidenced the presence of $\mathrm{Mn}$ accumulation in the magnetosomes synthesized by mixed populations of uncultured bacteria exposed to $\mathrm{MnCl}_{2}{ }^{26}$ They detected up to 2.81 atomic \% Mn by elemental analysis of thin sections of the synthesized magnetosomes. Later, Tanaka et al. analyzed the Mn doping of Magnetospirillum magneticum AMB1 grown in a $\mathrm{Mn}$-supplemented medium, reporting values of 2.7 atomic \% Mn. ${ }^{16}$ In the same way, Prozorov et al. and Pérez Gonzalez et al. presented a detailed characterization of magnetosomes in Magnetospirillum gryphiswaldense MSR-1 cells, grown in a Mn-containing medium, reaching values of 1 atomic \% Mn cations. ${ }^{20,21}$ These studies evidenced the tuning of the magnetic properties of the doped magnetosomes as a consequence of $\mathrm{Mn}$ incorporation by a reduction of the coercivity, an enhancement of the magnetic susceptibility, and a shift to lower temperatures of the Verwey transition, a fingerprint of magnetite. However, some key aspects as the oxidation state and coordination of the $\mathrm{Mn}$ in the doped magnetosomes and the origin of the resulting magnetic behavior still remain largely unknown.

In this study, we address the Mn doping of magnetosomes synthesized by M. gryphiswaldense MSR-1. M. gryphiswaldense forms cubo-octahedral magnetite nanoparticles with an average diameter of $\approx 40 \mathrm{~nm}$ and a narrow size distribution of $\approx 8 \mathrm{~nm}$. By the combination of structural and magnetic characterization techniques such as transmission electron microscopy (TEM) with energy-dispersive X-ray spectroscopy (EDX), X-ray absorption near-edge structure (XANES), and X-ray magnetic circular dichroism (XMCD), we evidence the presence of $\mathrm{Mn}$ (up to 2.3 atomic \%) in the magnetite structure. Our measurements suggest that $\mathrm{Mn}$ cations are incorporated as a combination of $\mathrm{Mn}^{2+}$ and $\mathrm{Mn}^{3+}$ preferably occupying tetrahedral and octahedral sites, respectively. The incorporation of Mn strongly influences the magnetic behavior of the synthesized magnetosomes. Theoretical modeling of the hysteresis loops of the $\mathrm{Mn}$-doped magnetosome chains with a modified Stoner-Wohlfarth approach sheds light on the different anisotropy contributions and their temperature dependence.

\section{EXPERIMENTAL SECTION}

Bacterial Strain and Growth Conditions. M. gryphiswaldense MSR-1 (DMSZ 6631) was grown in a modified flask standard medium ${ }^{27}$ in three-fourth 1 L-bottles loosely capped at $28{ }^{\circ} \mathrm{C}$ without shaking for $120 \mathrm{~h}$, after which well-formed magnetosomes were observed. Prior to the inoculation, the growth medium was supplemented with different amounts of $\mathrm{Fe}$ and $\mathrm{Mn}$ as $\mathrm{Fe}$ (III) and $\mathrm{Mn}$ (II)-citrates, respectively. Three samples were produced: (i) undoped magnetosomes (100 $\mu \mathrm{M}$ $\mathrm{Fe}(\mathrm{III})$-citrate), (ii) $\mathrm{Mn}(100)(10 \mu \mathrm{M} \mathrm{Fe}$ (III)-citrate and 100 $\mu \mathrm{M} \mathrm{Mn}(\mathrm{II})$-citrate), and (iii) $\mathrm{Mn}(480)$ magnetosomes (10 $\mu \mathrm{M} \mathrm{Fe}(\mathrm{III})$-citrate and $480 \mu \mathrm{M} \mathrm{Mn}$ (II)-citrate).

Magnetosomes have been measured either in the whole cells or isolated from the bacteria.

For the preparation of whole cell samples, the cells were harvested by centrifugation, fixed in $2 \%$ glutaraldehyde, and washed three times in $\mathrm{mQ}$ water and concentrated up to $10^{8}$ cell $/ \mathrm{mL}$.

Complementarily, magnetosomes were isolated from the bacteria following the protocol described by Grünberg et al. ${ }^{28}$ with minor modifications. The cells were collected by centrifugation, suspended in $20 \mathrm{mM}$ HEPES-4 mM EDTA $(\mathrm{pH}=7.4)$, and disrupted using a French press $(P=1.4 \mathrm{kbar})$. The lysated cells were sonicated to promote the separation of magnetosomes and centrifuged at $600 \mathrm{~g}$ for $5 \mathrm{~min}$ to remove cell debris. Then, magnetosomes were collected from the supernatant by magnetic separation and rinsed 10 times with $10 \mathrm{mM}$ HEPES-200 mM NaCl $(\mathrm{pH}=7.4)$. Finally, the isolated magnetosomes were redispersed in ultra-pure water.

Transmission Electron Microscopy (TEM), EnergyDispersive X-ray Spectroscopy (EDX), and High-Resolution TEM (HRTEM). TEM images were obtained on unstained cells and isolated magnetosomes adsorbed onto 300 mesh carbon-coated $\mathrm{Cu}$ grids with a Philips CM200 electron microscope that includes an EDX detector at an accelerating voltage of $200 \mathrm{kV}$. The particle-size distribution of the isolated magnetosomes was analyzed using a standard software for digital electron microscope image processing, Image J. ${ }^{29}$ EDX spectra were acquired with a counting time of 5 min to optimize the signal-to-noise ratio while minimizing the induced irradiation damage. The employed beam size was $\sim 65$ $\mathrm{nm}$, allowing the microanalysis of small clusters of several magnetosomes. Five spectra were acquired and averaged for each sample.

HRTEM measurements were carried out using a FEI Titan Cubed G2 60-300 electron microscope at $300 \mathrm{kV}$ equipped with a high-brightness X-FEG Schottky field emission electron gun, a monochromator, and CEOS GmbH spherical aberration (Cs) corrector on the image side. The structural parameters of the magnetosomes were obtained by Fourier transform analysis.

X-ray Absorption Near-Edge Structure (XANES). XANES measurements were performed on isolated magnetosomes to maximize the signal-to-noise ratio. For these measurements, magnetosomes were freeze-dried and thoroughly mixed with boron nitride, the resultant mixture was compacted into $5 \mathrm{~mm}$ diameter pellets.

Measurements were performed at room temperature and atmospheric conditions at the branch A of BM25-SpLine of the ESRF synchrotron facility (France). ${ }^{30} \mathrm{Fe}$ and $\mathrm{Mn}$ foils were measured to calibrate the energy at the beginning and end of the experiment. In these conditions, the edge position of the sample can be determined with an accuracy of $\Delta E=0.3 \mathrm{eV}$. Data for undoped magnetosomes (pure $\mathrm{Fe}_{3} \mathrm{O}_{4}$ ) and various $\mathrm{Mn}$ references were acquired in transmission mode ( $\mathrm{Fe}$ and/or Mn K-edges). Mn(100) magnetosome spectra were acquired in transmission mode at the $\mathrm{Fe} \mathrm{K}$-edge and in fluorescence yield mode at the Mn K-edge. A number of three to five spectra were acquired and averaged for each sample to improve the signal-to-noise ratio. All data were treated using Athena software from the Iffefit package. ${ }^{31}$

X-ray Magnetic Circular Dichroism (XMCD). Room temperature XMCD experiments were performed using the ALICE station ${ }^{32,33}$ at the PM3 beamline of BESSY II in Berlin, Germany. Data acquisition was achieved in transmission mode. A drop of $5 \mu \mathrm{L}$ of purified magnetosomes in an aqueous solution was deposited onto 300 mesh carbon-coated $\mathrm{Cu}$ grids. The concentration of the magnetosome solution was optimized for the proper detection of $\mathrm{Fe}$ and $\mathrm{Mn}$ ions at 

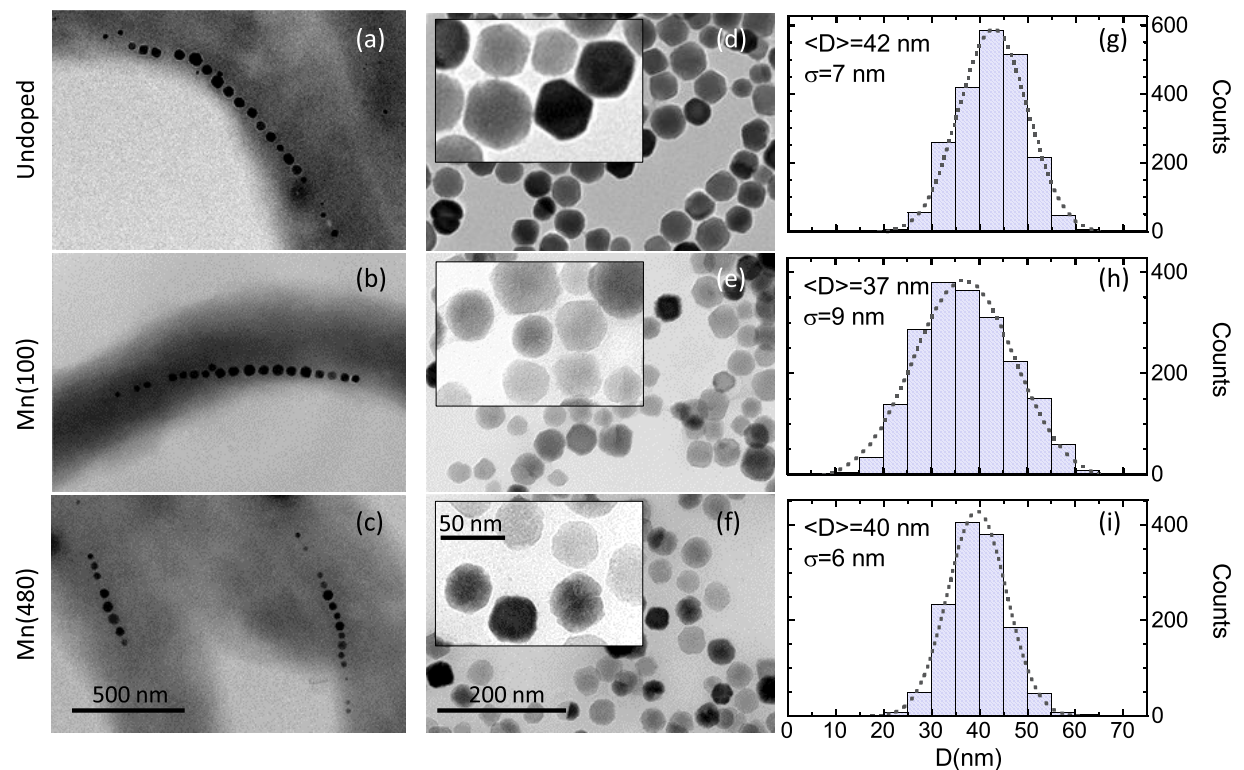

Figure 1. Representative TEM images of $(\mathrm{a}-\mathrm{c})$ M. gryphiswaldense and $(\mathrm{d}-\mathrm{f})$ isolated magnetosomes. Insets correspond to zoom-in images so as to highlight the observed morphological changes. $(\mathrm{g}-\mathrm{i})$ Size distribution for the undoped, $\mathrm{Mn}(100)$, and $\mathrm{Mn}(480)$ magnetosomes.
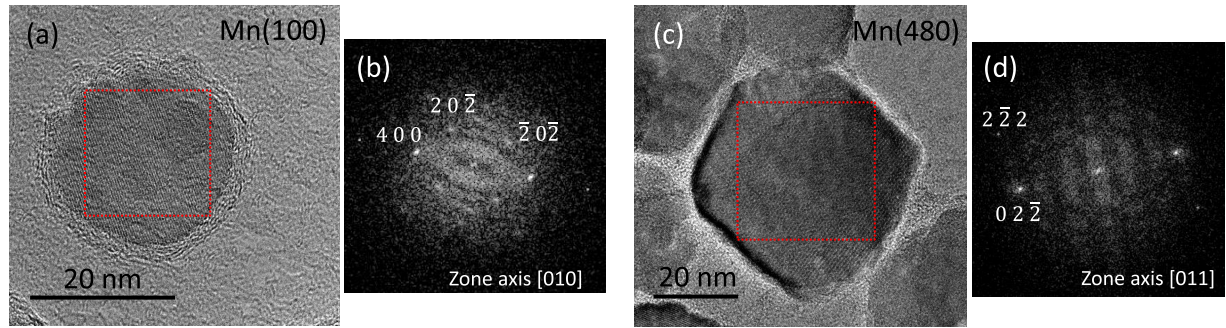

Figure 2. (a, c) HRTEM images of $\mathrm{Mn}(100)$ and $\mathrm{Mn}(480)$ magnetosomes and (b, d) Fourier transform images of isolated magnetosomes (red dotted squares).

their respective $\mathrm{L}_{3,2}$-edges: 0.2 and $0.5 \mathrm{mg} / \mathrm{mL} \mathrm{Fe}_{3} \mathrm{O}_{4}$ were used for the study of the undoped and Mn-doped magnetosomes at the $\mathrm{Fe} \mathrm{L}_{3,2}$-edges, respectively, while a $10 \mathrm{mg} / \mathrm{mL}$ $\mathrm{Fe}_{3} \mathrm{O}_{4}$-concentrated solution was used for the data acquisition at the $\mathrm{Mn} \mathrm{L}_{3,2}$-edges. X-ray radiation (circularly polarized, left helicity) impinged the sample surface at normal incidence. Xray absorption spectra $(I)$ were obtained across the $\mathrm{Fe}$ and $\mathrm{Mn}$ $\mathrm{L}_{3,2}$-edges with a step size of $0.2 \mathrm{eV}$ with an applied magnetic field parallel to the X-ray beam of $+0.35 \mathrm{~T}\left(I^{+}\right)$and $-0.35 \mathrm{~T}$ $\left(I^{-}\right)$. Three spectra were acquired and averaged to improve the signal-to-noise ratio. XMCD was defined as $\left(I^{+}-I^{-}\right)$.

Theoretical spectra at the $\mathrm{Mn} \mathrm{L}_{3,2}$-edge were simulated considering tetrahedral and octahedral environments for $\mathrm{Mn}^{2+}$ and octahedral occupancy for $\mathrm{Mn}^{3+}$ with the spin orbit coupling acting on the $3 \mathrm{~d}$ shell, the Slater integrals were scaled to $\kappa=80 \%$. The crystal-field parameter $10 \mathrm{Dq}$ was fixed to 1.56 $\mathrm{eV}$ for octahedral sites and $-0.6 \mathrm{eV}$ for tetrahedral ones. ${ }^{34}$ The calculated spectra were convoluted by a Lorentzian profile with a half-width half-maximum (HWHM) of $\Gamma_{\mathrm{L}_{3}=} 0.12 \mathrm{eV}$ and $\Gamma_{\mathrm{L}_{2}=}$ $0.24 \mathrm{eV}$, to account for the intrinsic core-hole lifetime broadening, and a Gaussian function, $\sigma=0.15 \mathrm{eV} \mathrm{HWHM}$, to account for the instrumental broadening. Spectra were simulated using the CTM4XAS program. ${ }^{35}$

Magnetic Measurements. The magnetic characterization was carried out on the freeze-dried cells. For that, the fixed and washed cells were highly concentrated and rapidly frozen by immersing them into liquid nitrogen. The frozen sample was immediately placed into a Telstar LyoQuest-85 plus freezedryer set at $-85^{\circ} \mathrm{C}$. The pressure and temperature control ensure the drying of the sample by sublimation, preserving the physical form of cells. The resulting powder sample was encapsulated in a gelatin capsule, giving rise to a structurally and magnetically isotropic sample where magnetosome chains are randomly oriented. ${ }^{36}$ Magnetic measurements were carried out in a superconducting quantum interference device magnetometer (Quantum Design MPMS-3). Isothermal magnetization loops were measured between $\mu_{0} H=+1$ and $-1 \mathrm{~T}$ from $T=5-300 \mathrm{~K}$ after a cooling process with either no applied magnetic field [zero-field-cooling (ZFC)-M(H)] or under an applied magnetic field of $1 \mathrm{~T}$ [field-cooling FC$M(H)]$.

\section{RESULTS AND DISCUSSION}

Structural and Morphological Characterization. Figure $1 \mathrm{a}-\mathrm{f}$ shows representative TEM images of $M$. gryphiswaldense and isolated magnetosomes for undoped, $\mathrm{Mn}(100)$, and $\mathrm{Mn}(480)$ samples. There are no significant differences in the cell morphology and chain arrangement between samples. The three cultures exhibit magnetosomes arranged in a single chain with a variable number of magnetosomes per cell between 10 and 30 (see the Supporting Information, Figure S1).

Figure $1 \mathrm{~g}-\mathrm{i}$ shows the particle size distributions determined from TEM images of the isolated magnetosomes, analyzing at least 1500 particles per sample to get reasonable statistics. The 

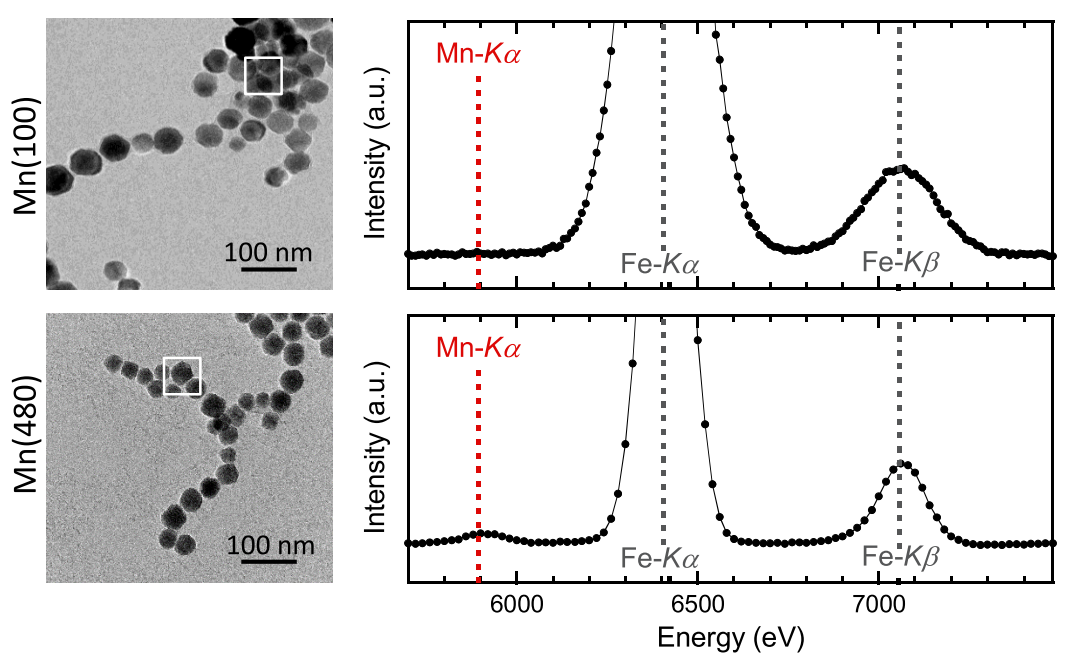

Figure 3. EDX spectra carried out on small clusters of $\mathrm{Mn}(100)$ and $\mathrm{Mn}(480)$ magnetosomes. The left panel presents TEM micrographs of magnetosome clusters in the region of interest. White squares delimit one out of the five EDX analyzed regions (beam size $65 \mathrm{~nm}$ ). The right panel displays the average EDX spectra of the five single regions. Vertical dashed lines indicate the position of $\mathrm{Mn} \mathrm{K} \alpha$, $\mathrm{Fe} \mathrm{K} \alpha$, and $\mathrm{Fe} \mathrm{K} \beta$ emission lines, respectively.
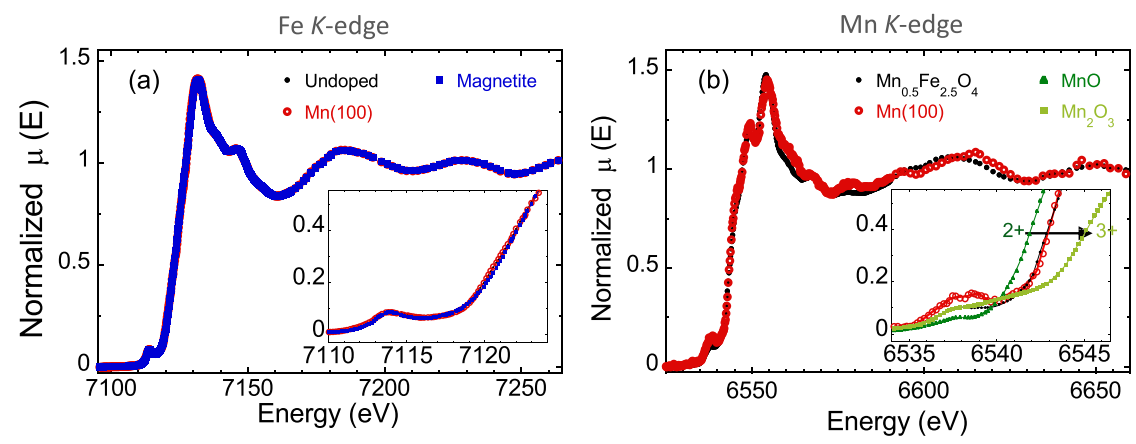

Figure 4. (a) Normalized Fe K-edge XANES spectra of undoped, $\mathrm{Mn}(100)$ magnetosomes, and bulk magnetite. The inset displays the pre-edge region in a more detailed depiction. (b) Normalized Mn K-edge XANES spectra of $\mathrm{Mn}(100)$ magnetosomes and $\mathrm{Mn}_{0.5} \mathrm{Fe}_{2.5} \mathrm{O}_{4}$. The inset is a zoom-in of the $\mathrm{Mn}$ pre-edge region compared to commercial oxides: $\mathrm{MnO}\left(\mathrm{Mn}^{2+}\right)$ and $\mathrm{Mn}_{2} \mathrm{O}_{3}\left(\mathrm{Mn}^{3+}\right)$. The Fe and Mn K-edge pre-edge regions of all samples have been fitted (continuous lines) considering a set of Gaussian profile peaks (see the Supporting Information).

resulting histograms were fitted by a Gaussian function. ${ }^{37,38}$ The undoped sample presents uniformly sized magnetosomes, as reflected in its corresponding histogram, where the size distribution is centered at $\langle D\rangle=42 \mathrm{~nm}$ with a standard deviation $\sigma=7 \mathrm{~nm} . \mathrm{Mn}(100)$ and $\operatorname{Mn}(480)$ magnetosomes present size distributions slightly shifted to lower values compared to the undoped magnetosomes. The size distribution of the $\operatorname{Mn}(100)$ peaks is centered at $\langle D\rangle=37 \mathrm{~nm}(\sigma=9$ $\mathrm{nm})$, while the distribution of the $\operatorname{Mn}(480)$ magnetosomes is centered at $\langle D\rangle=40 \mathrm{~nm}(\sigma=6 \mathrm{~nm})$.

While previous reports on $\mathrm{Mn}$ doping of magnetosomes do not show any significant differences in the morphology or size, ${ }^{16,20,21,26}$ TEM images presented in Figure 1 suggest certain morphological variations between samples. In particular, Mn-doped magnetosomes present more irregular edges as compared to the sharply faceted undoped particles.

Aiming to analyze the microstructural properties of the Mndoped magnetosomes, high-resolution TEM (HRTEM) imaging was performed. Figure 2 shows a single particle of $\mathrm{Mn}(100)$ and $\mathrm{Mn}(480)$ together with the Fourier transform image of the marked region. Both samples manifest a set of well-defined lattice fringes. From the indexing of the diffraction spots observed in the Fourier transform image, we determined the crystalline structure of both nanoparticles. $\mathrm{Mn}(100)$ and
$\mathrm{Mn}(480)$ magnetosomes evidence a magnetite structure oriented in the [010] and the [011] zone axis, respectively. It indicates that the cubic spinel structure of the magnetite is not disturbed upon doping of $\mathrm{Mn}$ ions.

The chemical composition of isolated Mn magnetosomes was evaluated by means of energy-dispersive X-ray spectroscopy (EDX) in TEM mode. Figure 3 displays TEM micrographs of the extracted magnetosomes and the EDX spectra obtained by averaging five individual regions of the doped samples. Both samples show two main peaks attributed to the dominant presence of iron in the magnetosomes. The one at lower energies $(\sim 6400 \mathrm{eV})$ corresponds to the Fe K $\alpha$ emission line and the one at higher energies $(\sim 7058 \mathrm{eV})$ to the $\mathrm{Fe} \mathrm{K} \beta$. The position of the $\mathrm{Mn} \mathrm{K} \alpha$ emission line $(\sim 5899 \mathrm{eV})$ has been marked with a red dashed line in the experimental spectra. No evidence of $\mathrm{Mn}$ has been found in the $\mathrm{Mn}(100)$ sample indicating that the presence of such an element is below the resolution limit of the technique ( $\approx 1$ atomic $\%)$. An average value of 2.3 atomic \% $\mathrm{Mn}$ was detected in the $\mathrm{Mn}(480)$ sample.

The coordination environment of the $\mathrm{Fe}$ and $\mathrm{Mn}$ ions in the magnetite structure has been investigated by means of X-ray absorption near-edge structure (XANES) and X-ray magnetic circular dichroism (XMCD) spectroscopies. XANES and 

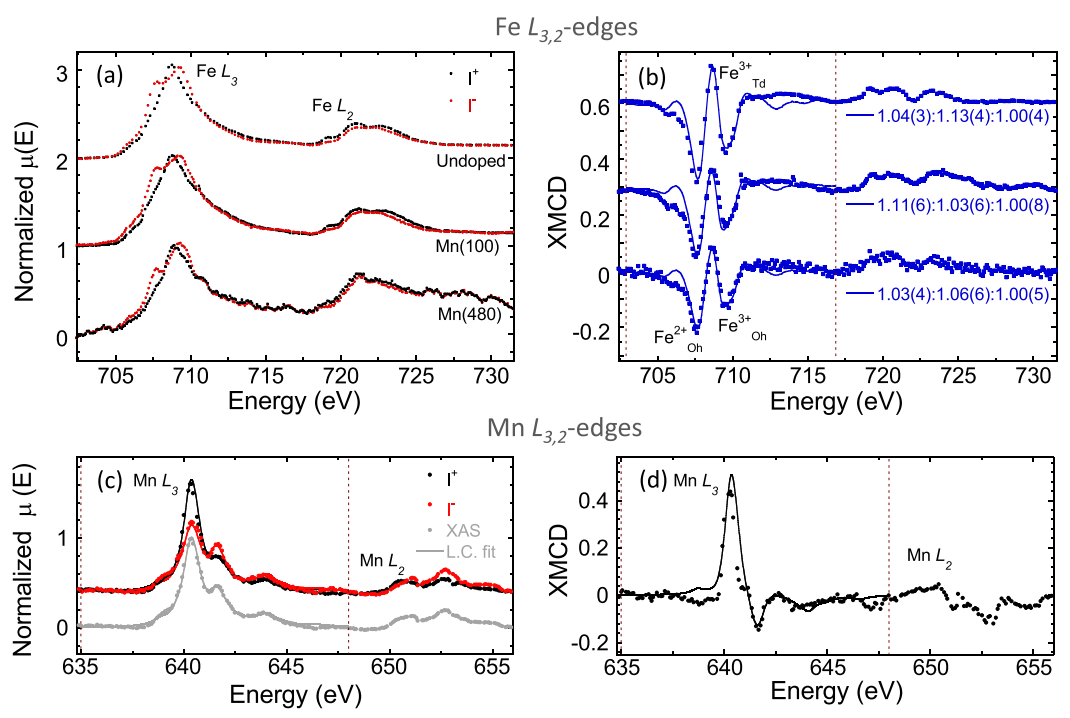

Figure 5. Transmission X-ray absorption spectra (XAS) acquired at $\pm 0.35 \mathrm{~T}\left(I^{ \pm}\right)$at the (a) Fe $\mathrm{L}_{3,2}$-edges of undoped, $\mathrm{Mn}(100)$, and $\mathrm{Mn}(480)$ magnetosomes and (c) $\mathrm{Mn} \mathrm{L}_{3,2}$-edges of $\mathrm{Mn}(480)$ magnetosomes. Spectra have been normalized by the peak intensity at the $\mathrm{L}_{3}$-edge of the nonmagnetic contribution of the XAS $\left(I^{+}+I^{-}\right) / 2$. The magnetic contributions (XMCD) given by $\left(I^{+}-I^{-}\right)$, are presented in $(\mathrm{b}, \mathrm{d})$. The continuous line presents the best theoretical simulation of the $\mathrm{Fe}_{3}$-edge XMCD spectra and $\mathrm{Mn}_{3}$-edge XAS and XMCD spectra in the energy window delimited by the vertical dotted lines (see the Supporting Information).

XMCD are very powerful techniques that provide accurate information on the oxidation state and site occupancy of the Mn cations in the spinel structure of the magnetite-where the Fe ions occupy three different sites: $8 \mathrm{Fe}^{2+}$ in octahedral sites $\left(O_{\mathrm{h}}\right), 8 \mathrm{Fe}^{3+}$ in tetrahedral $\left(T_{\mathrm{d}}\right)$ sites, and $8 \mathrm{Fe}^{3+}$ in octahedral $\left(O_{\mathrm{h}}\right)$ ones.

Figure 4a shows the Fe K-edge XANES spectra of undoped and $\mathrm{Mn}(100)$ magnetosomes together with bulk magnetite. As previously reported by our group, undoped magnetosomes accurately reproduce the spectrum of magnetite, ${ }^{18,39,40}$ and so do $\mathrm{Mn}$-doped magnetosomes. This resemblance is also highlighted in the inset in Figure 4a, which exhibits an identical edge position and pre-edge feature of the three samples. This fact suggests an unperturbed coordination environment around the Fe-absorbing atoms in $\mathrm{Mn}(100)$ magnetosomes, agreeing with the imperceptible presence of Mn detected by EDX in this sample.

However, the presence of $\mathrm{Mn}$ in the magnetite structure is confirmed by the spectrum obtained at the Mn absorption edge, which exhibits a good signal-to-noise ratio. Figure $4 \mathrm{~b}$ shows the Mn K-edge XANES spectrum of the $\mathrm{Mn}(100)$ magnetosomes together with the one of $\mathrm{Mn}_{0.5} \mathrm{Fe}_{2.5} \mathrm{O}_{4}$ provided by Dr. Mazarío, which presents a cation distribution similar to Mn-ferrite. $^{41}$ At a first glance, we observe the similarity between both spectra indicating that $\mathrm{Mn}$ is introduced into the spinel structure of the magnetite.

By looking at the edge position and the pre-edge region we can infer the oxidation state and the symmetry of the $\mathrm{Mn}$ absorber atom. The inset in Figure $4 \mathrm{~b}$ highlights the lowenergy region of the $\mathrm{Mn}$ K-edge XANES spectrum of the $\mathrm{Mn}$ (100) magnetosomes together with that corresponding to $\mathrm{MnO}\left(\mathrm{Mn}^{2+}\right)$ and $\mathrm{Mn}_{2} \mathrm{O}_{3}\left(\mathrm{Mn}^{3+}\right)$ reference samples. The edge of the $\mathrm{Mn}(100)$ magnetosomes $(6543.3 \mathrm{eV})$ appears between the edge position of $\mathrm{MnO}(6542.4 \mathrm{eV})$ and $\mathrm{Mn}_{2} \mathrm{O}_{3}(6546.0$ $\mathrm{eV})$. For similar compounds in which the absorbing element presents the same symmetry, there exists a linear relationship between the redox state and edge position. ${ }^{42}$ In the present case, as will be shown later, the different $\mathrm{Mn}$ ions in the sample could appear in different symmetries increasing the error associated with the quantification of the redox analysis of the edge. ${ }^{43-46}$ A rough estimation taking into account that there is a total edge displacement of $3.6 \mathrm{eV}$ from $\mathrm{Mn}^{2+}$ to $\mathrm{Mn}^{3+}$ spectra in octahedral compounds and a $0.9 \mathrm{eV}$ shift occurring between the $\mathrm{Mn}(100)$ magnetosomes and $\mathrm{MnO}\left(\mathrm{Mn}^{2+}\right)$ edges indicates that there are a majority of $\mathrm{Mn}^{2+}(75 \%)$ cations coexisting with $\mathrm{Mn}^{3+}$ cations $(25 \%)$.

On the other hand, the intensity and position of the preedge region are also related to the redox state and symmetry of the absorber atom. The Mn K-edge XANES spectrum of the $\mathrm{Mn}(100)$ magnetosomes exhibits the pre-edge peak located around $5 \mathrm{eV}$ before the edge position of the $\mathrm{Mn}$ K-edge, see Figure $4 \mathrm{~b}$. The high intensity of the pre-edge feature provides evidence for noncentrosymmetric tetrahedral sites. ${ }^{42,43,47,48}$ Moreover, the multiple-peak pre-edge feature suggests a coexistence with centrosymmetric octahedral sites. ${ }^{43}$ The centroid position of the $\mathrm{Mn}(100)$ magnetosomes, obtained from the fit to a set of Gaussian functions (see the Supporting Information), is located between the centroid position of $\mathrm{MnO}$ $\left(\mathrm{Mn}^{2+}\right)$ and $\mathrm{Mn}_{2} \mathrm{O}_{3}\left(\mathrm{Mn}^{3+}\right)$, indicating a mixed valence state. However, the coexistence of the two oxidation states and symmetries proves cumbersome to obtain further quantitative information from the pre-edge region in the $\mathrm{Mn}(100)$ sample. We will use XMCD data to better clarify this point.

The site occupancy and oxidation state of $\mathrm{Mn}$ in the Mndoped magnetosomes have been analyzed by means of XMCD at the $\mathrm{Fe}$ and $\mathrm{Mn} \mathrm{L}_{3,2}$-edges. The $\mathrm{Fe}_{3,2}$-edges $\left(I^{ \pm}\right)$and the XMCD $\left(I^{+}-I^{-}\right)$spectra of the undoped, $\operatorname{Mn}(100)$, and $\mathrm{Mn}(480)$ magnetosomes are depicted in Figure 5a,b. XMCD spectra show three main peaks characteristic of magnetite. ${ }^{49,50}$ Those peaks are attributed to the different occupations of the Fe cations: the two negative peaks are mainly due to the octahedral occupation of $\mathrm{Fe}^{2+}$ and $\mathrm{Fe}^{3+}$ ions, while the positive peak between them is due to the $\mathrm{Fe}^{3+}$ ions placed in tetrahedral sites. The opposite sign of the peaks indicates the antiferromagnetic coupling between octahedral and tetrahedral sites in magnetite. From the experimental XMCD spectra we 


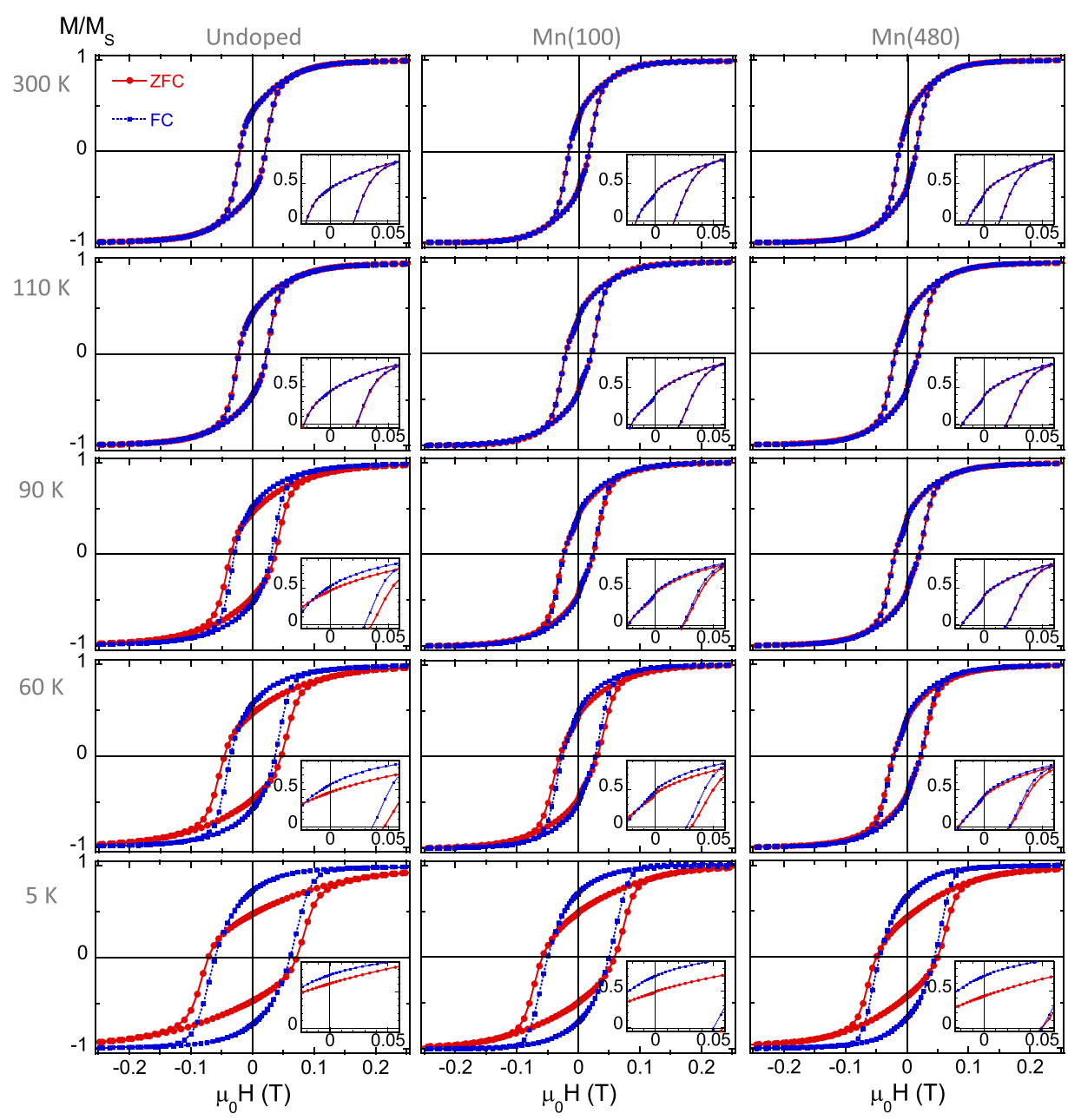

Figure 6. Comparison of experimental zero-field cooling (ZFC-M $(H))$ and field cooling (FC-M(H)) hysteresis loops of undoped, Mn(100), and $\mathrm{Mn}(480)$ magnetosomes at selected temperatures. The zoom-ins present more detailed depictions of the remanence region of the ZFC/FC- $M(H)$ loops where the kink is observed.

have determined the ratio $\mathrm{Fe}_{\mathrm{Oh}}^{2+} / \mathrm{Fe}_{\mathrm{Td}}^{3+} / \mathrm{Fe}_{\mathrm{Oh}}^{3+}$ by the linear combination fit of the theoretical spectra of the individual $\mathrm{Fe}$ components. The best linear combination fits of the $\mathrm{Fe}_{3}$-edge obtained between 703 and $717 \mathrm{eV}$ have been superimposed, as shown in Figure $5 \mathrm{~b}$. The computed ratios obtained were 1.04(3):1.13(4):1.00(5) for undoped, 1.11(6):1.03(6):1.00(8) for $\mathrm{Mn}(100)$, and 1.03(4):1.06(6):1.00(5) for $\mathrm{Mn}(480)$ magnetosomes. The three calculated ratios are close to the expected ratio for stoichiometric magnetite $(1: 1: 1){ }^{18}$ As previously inferred from the Fe K-edge XANES spectrum of $\mathrm{Mn}(100)$ magnetosomes, the incorporation of $\mathrm{Mn}$ cations barely alters the coordination environment of the Fe absorbing atoms.

Figure 5c,d displays Mn L-edge XAS $\left(I^{ \pm}\right)$and XMCD spectra of the $\mathrm{Mn}(480)$ magnetosomes, respectively. The $\mathrm{Mn}$ L-edge XAS and XMCD spectra corresponding to the $\mathrm{Mn}(100)$ sample can be found in the Supporting Information. We focused the analysis on the $\operatorname{Mn}(480)$ sample owing to the higher concentration of $\mathrm{Mn}$ in the sample and the better resolution of the $\mathrm{Mn} \mathrm{L}_{3,2}$-edges XAS and XMCD spectra. While the $M n(480)$ sample exhibits well-defined XAS and XMCD spectra, Mn(100) magnetosomes present less intense and noisier Mn L-edge peaks (see Figure S5 in the Supporting Information). Indeed, from the comparison of the intensity of the $\mathrm{Mn} \mathrm{L}_{3}$-edge XAS spectra of both samples, one can calculate the relative Mn concentration between them. From there we determined that the $\mathrm{Mn}$ concentration in the $\mathrm{Mn}(480)$ magnetosomes is $\approx 2.13$ higher than in the $\mathrm{Mn}(100)$ sample (see Table S3). This means that, since the $\mathrm{Mn}$ atomic percentage of $\mathrm{Mn}(480)$ magnetosomes determined by EDX was 2.3 atomic $\%, \mathrm{Mn}(100)$ rounds 1 atomic $\% \mathrm{Mn}$.

To quantify the site occupancies, the experimental Mn Ledge XAS and the XMCD spectra of the $\mathrm{Mn}(480)$ sample were modeled using ligand field multiplet calculations of the individual $\mathrm{Mn}$ sites. Theoretical XAS spectra have been calculated for $\mathrm{Mn}^{3+}$ and $\mathrm{Mn}^{2+}$ cations in both tetrahedral and octahedral coordinations (see the Supporting Information). The experimental nonmagnetic contribution of XAS $\left(\left(I^{+}\right.\right.$ $\left.+I^{-}\right) / 2$ ) was fitted to a linear combination of the three calculated spectra between 635 and $648 \mathrm{eV}$. The best linear combination fit has been superimposed, as shown in Figure $5 \mathrm{c}, \mathrm{d}$ and corresponds to $83.8(4) \% \mathrm{Mn}_{\mathrm{Td}}^{2+}, 4.0(4) \% \mathrm{Mn}_{\mathrm{Oh}}^{2+}$, and 12.2(3)\% $\mathrm{Mn}_{\mathrm{Oh}}^{3+}$.

The results obtained for the $\mathrm{Mn}(480)$ magnetosomes are in good agreement with those observed by XANES for the $\mathrm{Mn}(100)$ sample. Mn-doped magnetosomes present a dominant presence of $\mathrm{Mn}^{2+}$ cations occupying tetrahedral sites in coexistence with a minority of Mn cations, mostly $\mathrm{Mn}^{3+}$, in the octahedral coordination. The obtained percentages reproduce the inversion degree reported in the 
(a)

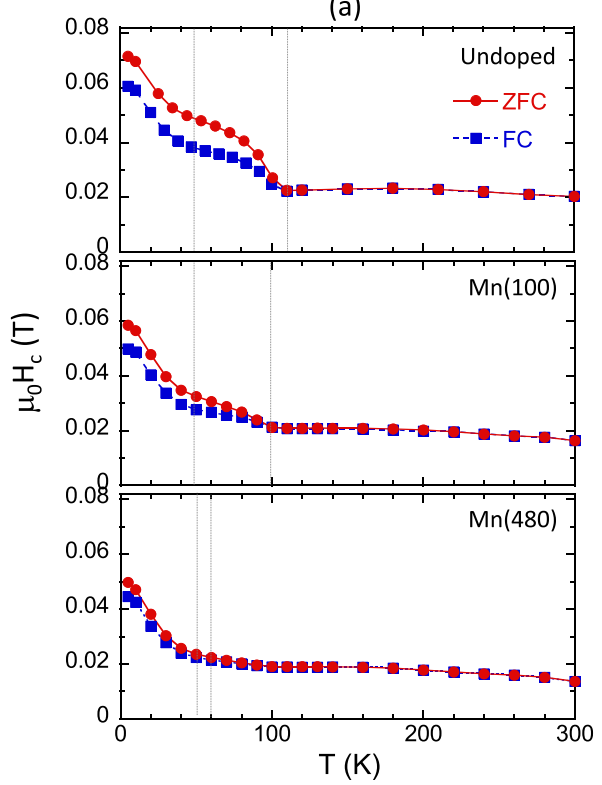

(c)

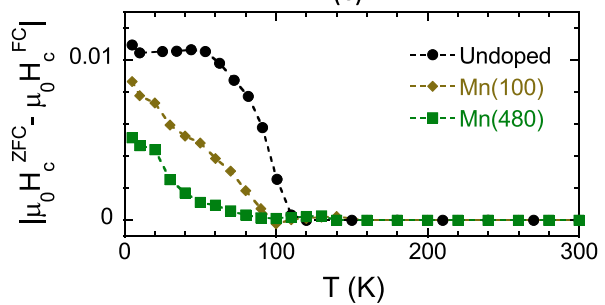

(b)

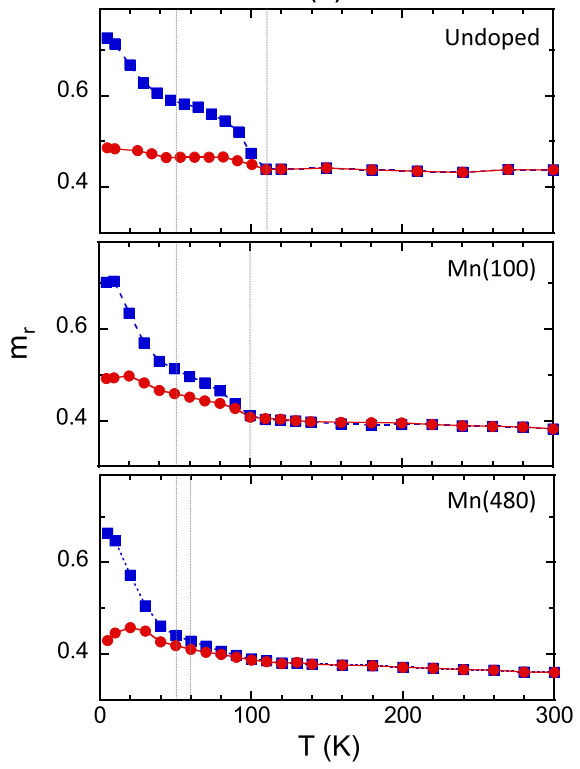

(d)

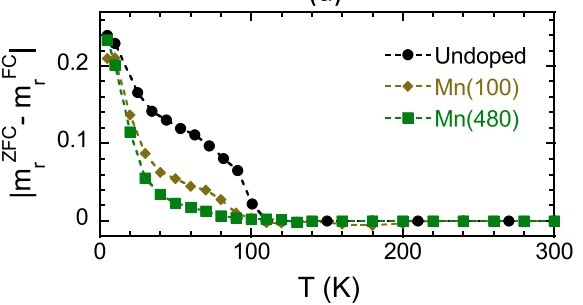

Figure 7. (a) Coercive field $\left(\mu_{0} H_{\mathrm{c}}\right)$ and (b) reduced remanence magnetization $\left(m_{\mathrm{r}}\right)$ evolution as a function of the temperature for the undoped, $\mathrm{Mn}(100)$, and $\mathrm{Mn}(480)$ magnetosomes. ZFC and FC-M(H) are compared. Temperature evolution of the difference (in absolute value) between ZFC and FC-M(H) in the (c) $\mu_{0} H_{\mathrm{c}}$ and (d) $m_{\mathrm{r}}$. Vertical dashed lines highlight the values at which the irreversibility between the ZFC and FC$\mathrm{M}(\mathrm{H})$ occurs: $107 \mathrm{~K}$ (undoped), $100 \mathrm{~K}(\mathrm{Mn}(100))$, and $60 \mathrm{~K}(\mathrm{Mn}(480))$, and the low temperature transition at $50 \mathrm{~K}$.

bibliography for bulk Mn-ferrite ${ }^{51-53}$ and ascribe the presence of $\mathrm{Mn}^{3+}$ cations to the inversion degree in Mn ferrites. ${ }^{24,41,52}$

Magnetic Measurements. To determine the role of $\mathrm{Mn}$ on the magnetic anisotropy of the doped magnetosomes, hereafter we present a complete magnetic characterization of undoped and Mn-doped magnetosomes as a function of temperature. All of the measurements have been carried out on the whole bacteria and hence on magnetosomes arranged in chains, where only intrachain interactions are expected.

Isothermal magnetization loops were measured after a cooling process with either no applied magnetic field (zerofield-cooling (ZFC)-M(H)) or under an applied magnetic field of $1 \mathrm{~T}$ (field-cooling (FC)- $M(H)$ ). ZFC/FC hysteresis loops of the three samples at selected temperatures are shown in Figure 6. In the case of the undoped sample, the ZFC and FC$M(H)$ curves perfectly overlap down to $T_{\mathrm{V}}=107 \mathrm{~K}$. At this temperature the well-known Verwey transition, involving a cubic-to-monoclinic crystallographic phase transition characteristic of magnetite, takes place. ${ }^{40,54,55}$ Below the Verwey temperature there is an important dependence on the thermal history; the $\mathrm{FC}-\mathrm{M}(H)$ shows a decrease of the coercivity combined with a noticeable increase of the remanence with respect to the ZFC-M $(H)$. In contrast, the ZFC/FC- $M(H)$ of $\mathrm{Mn}(100)$ and $\mathrm{Mn}$ (480) magnetosomes overlap down to 100 and $60 \mathrm{~K}$, respectively. Below these temperatures, differences between ZFC and FC-M(H) emerge. Furthermore, clear changes in the shape of the loops are observed between the samples. Contrary to the undoped magnetosomes, the ZFC/ FC- $M(H)$ curves of $\mathrm{Mn}(100)$ and $\mathrm{Mn}(480)$ samples present a kink in the remanence region down to 100 and $60 \mathrm{~K}$, respectively. Below these temperatures, the kink disappears. The presence of a kink is commonly related to the existence of two magnetic phases ${ }^{56}$ but, and as will be shown later, the disappearance of the kink at low temperatures suggests a different origin. Finally, in comparison with the undoped magnetosomes, the ZFC/FC-M $(H)$ curves of $\mathrm{Mn}(100)$ and $\mathrm{Mn}(480)$ samples exhibit a reduction of the coercivity in the whole range of temperatures ( $\approx 25$ and $\approx 40 \%$, respectively), in agreement with previous reports in the bibliography. ${ }^{16,20,21}$

The thermal evolution of the coercive field $\left(\mu_{0} H_{\mathrm{c}}^{\mathrm{ZFC} / \mathrm{FC}}\right)$ and the reduced remanence magnetization $\left(m_{\mathrm{r}}^{\mathrm{ZFC} / \mathrm{FC}}=\left(M_{\mathrm{r}}\right)\right.$ $\left.M_{\mathrm{s}}\right)^{\mathrm{ZFC} / \mathrm{FC}}$ ) of the three samples can be easily tracked in Figure 7. For the undoped sample, both ZFC and FC-M $(H)$ present a nearly constant coercivity from $300 \mathrm{~K}$ down to $T_{\mathrm{V}}$ $\left(\mu_{0} H_{\mathrm{c}}^{\mathrm{ZFC} / \mathrm{FC}} \approx 22 \mathrm{mT}\right)$. Below the Verwey transition, the sample presents a marked irreversibility between $\mu_{0} H_{c}^{\mathrm{ZFC}}$ and $\mu_{0} H_{\mathrm{c}}^{\mathrm{FC}} \cdot \mu_{0} H_{\mathrm{c}}^{\mathrm{ZFC}}$ increases up to $48 \mathrm{mT}$ at $50 \mathrm{~K}$, when a second transition attributed to the ordering of electron spins in magnetite at low temperature occurs. ${ }^{57-59}$ Below that temperature, the coercive field steeply increases reaching values of $72 \mathrm{mT}$ at $5 \mathrm{~K}$. The FC-M $(H)$ exhibits similar but less intense features. Below the Verwey transition, $\mu_{0} H^{\mathrm{FC}}$ increases up to $39 \mathrm{mT}$ at $50 \mathrm{~K}$ and rises up to $60 \mathrm{mT}$ at $5 \mathrm{~K}$. On the other hand, $m_{\mathrm{r}}^{\mathrm{ZFC}}$ remains fairly constant between 0.44 and 
0.49 throughout the whole temperature range. These values around 0.5 indicate that the undoped magnetosomes behave like randomly oriented uniaxial magnetic single domains in the framework of the Stoner-Wohlfarth model. ${ }^{60}$ In contrast, when the field is applied in the cooling process, $m_{\mathrm{r}}^{\mathrm{FC}}$ increases abruptly below the Verwey transition up to 0.6 at $50 \mathrm{~K}$ and up to 0.72 at $5 \mathrm{~K}$.

The magnetic response of the $\mathrm{Mn}(100)$ and $\mathrm{Mn}(480)$ magnetosomes changes notably. The coercive fields of the ZFC/FC- $M(H)$ remain roughly constant down to $100 \mathrm{~K}$, for the $\mathrm{Mn}(100)$ magnetosomes $\left(\mu_{0} H_{\mathrm{c}}^{\mathrm{ZFC} / \mathrm{FC}} \approx 20 \mathrm{mT}\right)$, and $60 \mathrm{~K}$, in the case of the $\mathrm{Mn}(480)$ sample $\left(\mu_{0} \mathrm{H}_{\mathrm{c}}^{\mathrm{ZFC} / \mathrm{FC}} \approx 15 \mathrm{mT}\right)$. Below these temperatures, the splitting of the ZFC and FC$M(H)$ occurs. In the case of the $\mu_{0} H_{\mathrm{c}}^{\mathrm{ZFC}}$, a smooth increase down to $50 \mathrm{~K}$ takes place. Beneath the low-temperature transition, $\mu_{0} H_{\mathrm{c}}^{\mathrm{ZFC}}$ steeply rises up to 58 and $50 \mathrm{mT}$, respectively. Similarly, $\mu_{0} H_{\mathrm{c}}^{\mathrm{FC}}$ rises softly at $50 \mathrm{~K}$ followed by the increase up to $50 \mathrm{mT}(\mathrm{Mn}(100))$ and $44 \mathrm{mT}(\mathrm{Mn}(480))$ at $5 \mathrm{~K}$. On the other hand, the reduced remanence magnetizations of the $\mathrm{ZFC} / \mathrm{FC}-M(H)$ remain fairly constant down to $100 \mathrm{~K}\left(m_{\mathrm{r}}^{\mathrm{ZFC} / \mathrm{FC}} \approx 0.39\right)$ and $60 \mathrm{~K}\left(m_{\mathrm{r}}^{\mathrm{ZFC} / \mathrm{FC}} \approx 0.36\right)$, respectively. Below these temperatures, $m_{\mathrm{r}}^{\mathrm{ZFC}}$ increases gradually equating the values obtained for the undoped magnetosomes at low temperature. In the case of the FC$M(H), m_{\mathrm{r}}^{\mathrm{FC}}$ increases moderately down to $50 \mathrm{~K}$ and then rises up to 0.71 at $5 \mathrm{~K}$, for the $\mathrm{Mn}(100)$ sample, and 0.66 , in the case of $\mathrm{Mn}(480)$ magnetosomes.

The relative change of the coercive field and remanence magnetization between the ZFC and FC-M $(H)$ measurements as a function of temperature for the three samples can be tracked in Figures $7 \mathrm{c}$,d. Here, it is clear that the divergence between the ZFC and FC-M $(H)$ values decreases with increasing $\mathrm{Mn}$ doping. The irreversibility between the ZFC and FC-M $(H)$ seems to root its origin in the Verwey transition, as will be discussed in the following section. Hence, it suggests that the cubic-to-monoclinic crystallographic phase transition still occurs in the presence of Mn cations but it is shifted from $107 \mathrm{~K}$ (undoped magnetosomes) to $100 \mathrm{~K}$ for the $\mathrm{Mn}(100)$ sample, and down to $60 \mathrm{~K}$, for the $\mathrm{Mn}(480)$ magnetosomes. Similar results are inferred from the conventional ZFC and FC $M(T)$ curves presented in Figure $S 7$ in the Supporting Information and previously reported by Prozorov and coworkers. $^{21}$ It is worth noting that the temperature values at which the split occurs coincide with the ones in which the kink of the $M(H)$ curves becomes negligible.

Magnetic Model. Aiming to understand the role of $\mathrm{Mn}$ in the magnetic properties of the doped magnetosomes, with special focus on their magnetic anisotropy, we have carried out numerical simulations of the magnetization dynamics of the magnetosomes. These have been computed at different temperatures considering a Stoner-Wohlfarth model with some pertinent modifications, as described previously by our group. ${ }^{18,61,62}$ To achieve clear cut conclusions, we have selected the $\mathrm{Mn}(480)$ sample, which has evidenced more distinct magnetic differences compared to the undoped magnetosomes. Precisely, we just remind here that $\mathrm{Mn}(100)$ magnetosomes exhibit an intermediate behavior. All of the simulations presented here were performed considering a single magnetic phase.

In brief, magnetosome chains were considered as a collection of independent single domain nanoparticles large enough to be thermally stable and so to have the magnetization firmly anchored at the minimum energy states. Therefore, the physical problem can be implemented using a single particle approach described by Stoner-Wohlfarth. Interparticle dipolar interactions occurring between the nearest magnetosome neighbors in the chain are assumed to impose an additional anisotropy contribution, equal for all. ${ }^{63-65}$ Note that the chain-chain interactions in the freeze-dried cells are negligible due to the large distance separating two chains of magnetosomes. $^{36,66}$ The equilibrium orientation of each magnetic dipole is therefore calculated by minimizing the single dipole energy density, $E$, which is computed as the sum of three contributions: ${ }^{18,61}$

(i) The first term of the single dipole energy density is the cubic magnetocrystalline energy $\left(E_{\mathrm{c}}\right)$

$$
E_{\mathrm{c}}(\theta, \varphi)=K_{\mathrm{c}} \frac{\left[\sin ^{4}(\theta) \sin ^{2}(2 \varphi)+\sin ^{2}(2 \theta)\right]}{4}
$$

$K_{\mathrm{c}}$ being the cubic anisotropy constant and $\theta$ and $\varphi$ the polar and azimuthal angles in spherical coordinates, respectively, of the magnetic moment $\left(\hat{u}_{\mathrm{m}}\right)$ considering the $\langle 100\rangle$ crystallographic directions as the reference system (see the Supporting Information, Figure S8).

(ii) An effective uniaxial anisotropy contribution along the $\hat{u}_{\text {uni }}$ axis arising from the competition between the magnetosome shape anisotropy and the interparticle dipolar interactions between the closest magnetosome neighbors in the chain

$$
E_{\text {uni }}(\theta, \varphi)=K_{\text {uni }}\left[1-\left(\hat{u}_{\text {uni }} \cdot \hat{u}_{\mathrm{m}}\right)^{2}\right]
$$

where $K_{\text {uni }}$ is the uniaxial anisotropy constant. On the basis of the previously reported chain configuration of undoped magnetosomes in M. gryphiswaldense, and for the sake of simplicity, the direction of the uniaxial axis $\hat{u}_{\text {uni }}$ has been fixed at $25^{\circ}$ out of the chain axis (which corresponds to the [111] crystallographic easy axis of magnetite), toward the $\langle 110\rangle$ direction. ${ }^{61,67}$

(iii) The Zeeman energy $\left(E_{\text {Zeeman }}\right)$ in an external magnetic field $\mu_{0} \vec{H}$ given by

$$
E_{\text {Zeeman }}(\theta, \varphi)=-\mu_{0} M H\left(\hat{u}_{\mathrm{H}} \cdot \hat{u}_{\mathrm{m}}\right)
$$

where $\hat{u}_{\mathrm{H}}$ represents the external magnetic field unit vector.

Thus, the single dipole energy density $E$ is described as

$$
E(\theta, \varphi)=E_{\mathrm{c}}(\theta, \varphi)+E_{\text {uni }}(\theta, \varphi)+E_{\text {Zeeman }}(\theta, \varphi)
$$

Under these considerations, the ZFC/FC-M(H) loops at different temperatures have been simulated assuming a dynamical approach described elsewhere. ${ }^{61,68,69} K_{\mathrm{c}}$ and $K_{\text {uni }}$ have been adjusted to achieve the best match between the experiment and theory. $K_{\mathrm{c}}$ and $K_{\mathrm{uni}}$ values found in simulations are presented in Figure 9 and will be discussed hereafter. As shown in Figure $8 \mathrm{a}-\mathrm{d}$, the simulated curves accurately reproduce the experimental hysteresis loops of the $\mathrm{Mn}(480)$ magnetosomes including the observed kink present in the remanence region down to $60 \mathrm{~K}$. As shown in the Supporting Information, Figure S9, the presence or absence of this kink depends on the ratio $r$ between the absolute value of the cubic anisotropy constant and the uniaxial anisotropy constant, $r=\left|K_{\mathrm{c}}\right| /\left|K_{\text {uni }}\right|$, so that for $r>1$ a clear kink is observed, while for $r \leq 1$ the kink is not distinguished anymore. Contrary to the undoped magnetosomes, where $r \leq 1$ from $300 \mathrm{~K}$ to $T_{\mathrm{V}}{ }^{18}$ the $\mathrm{Mn}(480)$ magnetosomes show a ratio $r>1$ down to the Verwey transition, giving rise to the observed kink (see the 


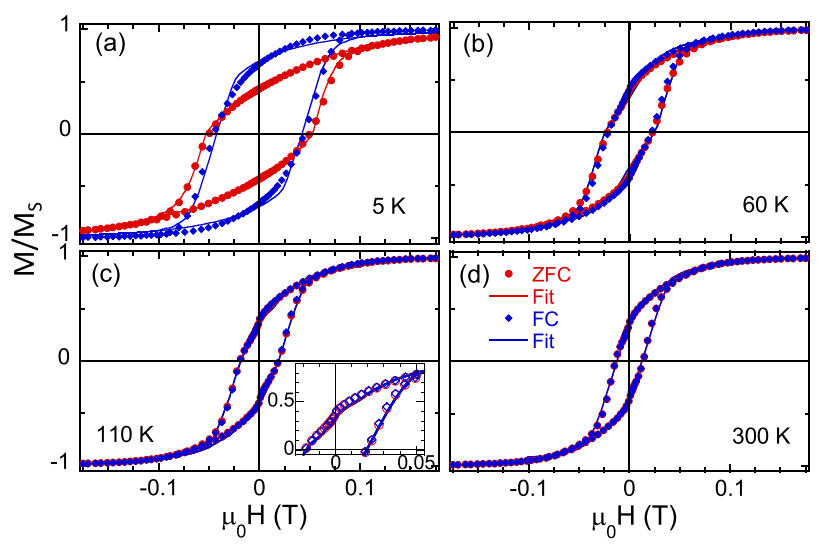

Figure 8. $\mathrm{ZFC} / \mathrm{FC}-\mathrm{M}(H)$ loops of the $\mathrm{Mn}(480)$ magnetosomes measured at (a) $5 \mathrm{~K}$, (b) $60 \mathrm{~K}$, (c) $110 \mathrm{~K}$, and (d) $300 \mathrm{~K}$. Continuous lines correspond to the simulated loops considering the anisotropy constants presented in Figure 9. The inset highlights the reproducibility of the kink at $110 \mathrm{~K}$.

Supporting lnformation Figure S10). As described hereafter, below the Verwey transition the anisotropy becomes essentially uniaxial, being the reason why the kink disappears below $T_{\mathrm{V}}$.

The thermal evolution obtained for $K_{\mathrm{c}}$ and $K_{\mathrm{uni}}$ for the $\mathrm{Mn}(480)$ magnetosomes, as well as for the undoped sample (extracted from Marcano et al. ${ }^{18}$ ) is shown in Figure 9.

From the simulation, we find that $K_{c}$ is negative from $300 \mathrm{~K}$ down to $60 \mathrm{~K}$. The absolute value of $K_{\mathrm{c}}\left(\left|K_{\mathrm{c}}\right|\right)$ increases from $10.80 \mathrm{~kJ} / \mathrm{m}^{3}$ at $300 \mathrm{~K}$ to $16.00 \mathrm{~kJ} / \mathrm{m}^{3}$ at $110 \mathrm{~K}$. Then, $\left|K_{\mathrm{c}}\right|$ slightly decreases down to $14 \mathrm{~kJ} / \mathrm{m}^{3}$ at $60 \mathrm{~K}$. Below this temperature the experimental $M(H)$ loops present an essentially uniaxial behavior and the role of the cubic magnetocrystalline anisotropy becomes negligible in the simulation. In comparison with the undoped magnetosomes, the incorporation of $\mathrm{Mn}^{2+, 3+}$ into the spinel structure of magnetite introduces an additional negative contribution to the cubic magnetocrystalline energy. The whole trend as well as the obtained anisotropy values of $K_{\mathrm{c}}$ agree with the results reported in the late fifties in single crystals of Mn-substituted magnetite considering a concentration of $\mathrm{Mn}$ around 3 atomic $\%^{70,71}$ On the other hand, $K_{\text {uni }}$ remains constant (slightly varying between 8 and $10 \mathrm{~kJ} / \mathrm{m}^{3}$ ) from the room temperature down to $60 \mathrm{~K}$. As mentioned above, $K_{\text {uni }}$ stems from the competition between the magnetosome shape anisotropy and the strength of the dipolar magnetic interactions, both depending only slightly on the temperature in this range. A similar tendency was observed for undoped magnetosomes from $300 \mathrm{~K}$ to $T_{\mathrm{V}}$, reporting values around $11-13 \mathrm{~kJ} /$ $\mathrm{m}^{3}{ }^{18,61,72}$ The reduction of the $\approx 25 \%$ observed in $K_{\text {uni }}$ arises from the competition of the two anisotropy terms: the shape anisotropy and the dipolar interactions. No substantial differences were observed in the size and the arrangement of the magnetosomes within the bacteria that might affect the dipolar magnetic interactions although slight variations on the magnetic moment of the magnetosomes may occur as a consequence of $\mathrm{Mn}$ doping. On the other hand, TEM images show a modification of the shape of the magnetosomes with $\mathrm{Mn}$ doping. The presence of softly faceted magnetosomes could alter the shape anisotropy, and hence, the $K_{\text {uni. }}$ Below 60 $\mathrm{K}, K_{\text {uni }}$ in the $\mathrm{Mn}(480)$ magnetosomes suddenly increases up to $27 \mathrm{~kJ} / \mathrm{m}^{3}$, also mimicking the trend of the undoped magnetosomes below $T_{\mathrm{V}}$. When the cubic-to-monoclinic transition takes place, the magnetocrystalline anisotropy of the undoped sample changes from cubic to essentially uniaxial along the $\langle 100\rangle$ crystallographic directions. Thus, the effective uniaxial anisotropy results from the competition of the magnetocrystalline anisotropy, shape anisotropy, and dipolar magnetic interactions with neighboring magnetosomes in the chain.

Finally, the differences observed between the FC and ZFC cases are attributed to the orientation distribution of the monoclinic axis below the Verwey transition. It is well known that cooling under an applied magnetic field triggers the unequivalency of the three $\langle 100\rangle$ monoclinic axes, setting the easy magnetization axis along that $\langle 100\rangle$ direction that is closer to the direction of the applied field. ${ }^{70}$ However, aiming to reproduce the experimental hysteresis loops of the magnetosomes at low temperature, we further need to assume an intrinsic discrimination of the three $\langle 100\rangle$ crystallographic directions. That is, in the absence of an applied field, the orthorhombic deformation would preferentially take place along the $\langle 100\rangle$ direction closer to the easy magnetization axis of each magnetosome $\left(\hat{u}_{\text {uni }}\right.$, which points $25^{\circ}$ out of the chain axis toward the $\langle 110\rangle) .^{61,67}$ In contrast, when an external field is applied in the cooling process, the three $\langle 100\rangle$ directions become equally probable. Thus, in the FC measurements, twothirds of the orthorhombic deformation will take place along the $\langle 100\rangle$ direction closer to $\hat{u}_{\text {uni }}$, and one-third will occur in the remaining $\langle 100\rangle$ direction. The latter is approximately

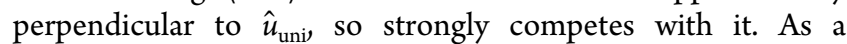
consequence, this gives rise to a decrease of the effective uniaxial anisotropy in the FC case with respect to the ZFC, which explains the experimentally observed decrease in the coercive field in the FC situation (see Figure 8 and the Supporting Information, Figure S11).
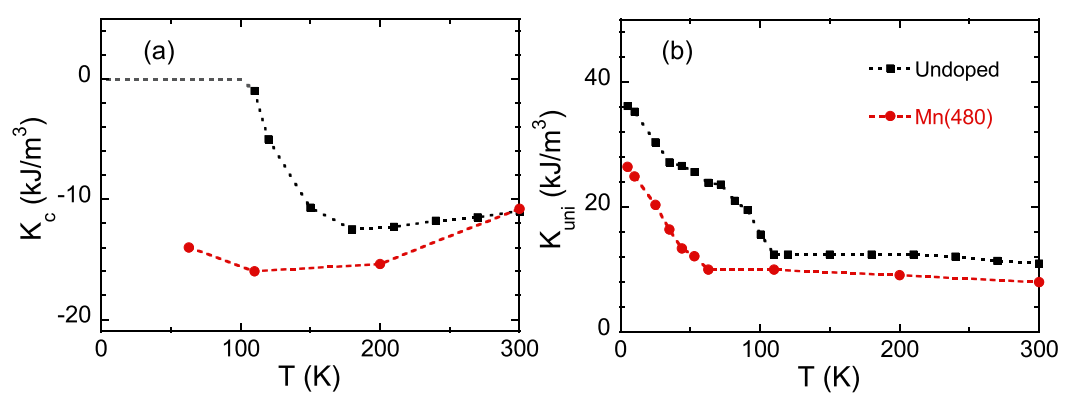

Figure 9. Best theoretical-experimental matching values for (a) cubic magnetocrystalline, $K_{\mathrm{c}}$ and (b) uniaxial anisotropy, $K_{\text {unii }}$ constants obtained for $\mathrm{Mn}(480)$ compared to undoped magnetosomes (extracted from Marcano et al. ${ }^{18}$ ). 


\section{CONCLUSIONS}

In conclusion, we carried out a complete study on the $\mathrm{Mn}$ doping of magnetosomes within M. gryphiswaldense MSR-1. Using two different culture media differing in the $\mathrm{Mn}$ concentration we have demonstrated the possibility to obtain Mn doping grade magnetosomes. This opens up the possibility to tune the magnetic properties under ambient conditions for a range of advanced biomedical applications.

From the combination of structural techniques we have detected up to 2.3 atomic \% $\mathrm{Mn}$ incorporated in the crystallographic structure of the magnetite, with a dominant presence of $\mathrm{Mn}^{2+}$ in the tetrahedral coordination coexisting with a minority of $\mathrm{Mn}$ ions, predominantly $\mathrm{Mn}^{3+}$, in the octahedral coordination. The Mn doping of the magnetosomes results in a notable change of their magnetic properties, which has been addressed by a complete magnetic characterization based on the study of the ZFC/FC-M $(H)$ loops as a function of temperature. Prominent among these are a reduction of the coercive field concomitant with a shift of the Verwey transition to lower temperatures with increasing Mn doping. On the basis of the slightly modified Stoner-Wohlfarth model and considering the different anisotropy contributions, we have been able to accurately reproduce the magnetic behavior of Mn-doped magnetosomes. This has allowed us to shed light on the role of $\mathrm{Mn}$ incorporation in the magnetocrystalline anisotropy of the magnetosomes, which accurately reproduced the behavior classically observed in single crystals of $\mathrm{Mn}$ substituted magnetites. Furthermore, we observed a decrease in the effective uniaxial anisotropy attributed to the modification of the shape of the doped magnetosomes, also suggested by TEM imaging.

\section{ASSOCIATED CONTENT}

\section{(s) Supporting Information}

The Supporting Information is available free of charge at https://pubs.acs.org/doi/10.1021/acs.jpcc.0c07018.

Analysis of the influence of $\mathrm{Mn}$ in the number of magnetosomes per chain; Fe K-edge XANES spectra comparison of bulk magnetite and maghemite with undoped and $\operatorname{Mn}(100)$ magnetosomes; pre-edge fits at $\mathrm{Fe}$ and $\mathrm{Mn} \mathrm{K}$-edges; determination of the relative $\mathrm{Mn}$ concentration between $\mathrm{Mn}(100)$ and $\mathrm{Mn}(480)$ magnetosomes from Mn L-edge XAS spectroscopy; details of XMCD simulations; zero-field cooling/field cooling (ZFC-FC)-M(H) curves; reference frame used in the magnetic simulations; role of the ratio $r=\left|K_{\mathrm{c}}\right| /\left|K_{\mathrm{uni}}\right|$; simulations of ZFC and FC-M(H) loops of undoped magnetosomes at $5 \mathrm{~K}$ (PDF)

\section{AUTHOR INFORMATION}

\section{Corresponding Author}

Lourdes Marcano - Helmholtz-Zentrum Berlin für Materialien und Energie, 12489 Berlin, Germany; Dpto. Electricidad y Electrónica, Universidad del País Vasco-UPV/EHU, 48940 Leioa, Spain; 이이이.org/0000-0001-9397-6122;

Email: lourdes.marcano@ehu.eus

\section{Authors}

Iñaki Orue - SGIker, Universidad del Páls Vasco-UPV/EHU, 48940 Leioa, Spain

Ana García-Prieto - Departmento Física Aplicada I, Universidad del País Vasco-UPV/EHU, 48013 Bilbao,
Spain; BCMaterials, Bld. Martina Casiano 3rd floor, 48940 Leioa, Spain

Radu Abrudan - Helmholtz-Zentrum Berlin für Materialien und Energie, 12489 Berlin, Germany

Javier Alonso - CITIMAC, Universidad de Cantabria, 39005 Santander, Spain; 이이이.org/0000-0003-0045-5390

Luis Fernández Barquín - CITIMAC, Universidad de Cantabria, 39005 Santander, Spain

Sergio Valencia - Helmholtz-Zentrum Berlin für Materialien und Energie, 12489 Berlin, Germany; 이이.org/00000002-3912-5797

Alicia Muela - Departmento Inmunología, Microbiología y Parasitología, Universidad del Pás Vasco-UPV/EHU, 48940 Leioa, Spain; BCMaterials, Bld. Martina Casiano 3rd floor, 48940 Leioa, Spain

M. Luisa Fdez-Gubieda - Dpto. Electricidad y Electrónica, Universidad del País Vasco-UPV/EHU, 48940 Leioa, Spain; BCMaterials, Bld. Martina Casiano 3rd floor, 48940 Leioa,

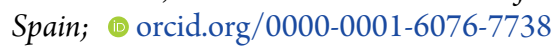

Complete contact information is available at:

https://pubs.acs.org/10.1021/acs.jpcc.0c07018

\section{Notes}

The authors declare no competing financial interest.

\section{ACKNOWLEDGMENTS}

L.M. acknowledges the financial support provided through a postdoctoral fellowship from the Basque Government (POS2019-2-0017). The Spanish and Basque Governments are acknowledged for funding under project numbers MAT201783631-C3-R and IT-1245-19, respectively. We acknowledge the technical and human support provided by SGIker (UPV/ EHU). We thank the ESRF (CRG BM25 beamline-SpLine) and HZB for the allocation of synchrotron radiation beamtime and funding under the project CALIPSOplus (Grant Agreement 730872) from the EU Framework Program for Research and Innovation HORIZON2020. We thank A. Serrano for her assistance in the XANES measurements.

\section{REFERENCES}

(1) Bazylinski, D. A.; Frankel, R. B. Magnetosome formation in prokaryotes. Nat. Rev. Microbiol. 2004, 2, 217-230.

(2) Bazylinski, D. A.; Lefêvre, C. T.; Schüler, D. The Prokaryotes; Rosenberg, E.; DeLong, E. F.; Lory, S.; Stackebrandt, E.; Thompson, F., Eds.; Springer Berlin Heidelberg: Berlin, Heidelberg, 2013; pp 453-494.

(3) Bazylinski, D. A.; Lefêvre, C. T.; Lower, B. H. Nanomicrobiology; Barton, L. L.; Bazylinski, D. A.; Xu, H., Eds.; Springer: New York, 2014; Chapter 3, pp 39-74.

(4) Uebe, R.; Schüler, D. Magnetosome biogenesis in magnetotactic bacteria. Nat. Rev. Microbiol. 2016, 14, 621-637.

(5) Alphandéry, E. Applications of Magnetosomes Synthesized by Magnetotactic Bacteria in Medicine. Front. Bioeng. Biotechnol. 2014, 2, 5.

(6) Muela, A.; Muñoz, D.; Martín-Rodríguez, R.; Orue, I.; Garaio, E.; Abad Díaz de Cerio, A.; Alonso, J.; García, J. Á.; Fdez-Gubieda, M. L. Optimal Parameters for Hyperthermia Treatment Using Biomineralized Magnetite Nanoparticles: Theoretical and Experimental Approach. J. Phys. Chem. C 2016, 120, 24437-24448.

(7) Sun, J.; Tang, T.; Duan, J.; Xu, P.-x.; Wang, Z.; Zhang, Y.; Wu, L.; Li, Y. Biocompatibility of bacterial magnetosomes: Acute toxicity, immunotoxicity and cytotoxicity. Nanotoxicology 2010, 4, 271-283.

(8) Mannucci, S.; Ghin, L.; Conti, G.; Tambalo, S.; Lascialfari, A.; Orlando, T.; Benati, D.; Bernardi, P.; Betterle, N.; Bassi, R.; et al. 
Magnetic Nanoparticles from Magnetospirillum gryphiswaldense Increase the Efficacy of Thermotherapy in a Model of Colon Carcinoma. PLoS One 2014, 9, No. e108959.

(9) Orlando, T.; Mannucci, S.; Fantechi, E.; Conti, G.; Tambalo, S.; Busato, A.; Innocenti, C.; Ghin, L.; Bassi, R.; Arosio, P.; et al. Characterization of magnetic nanoparticles from Magnetospirillum gryphiswaldense as potential theranostics tools: Characterization of Magnetic Nanoparticles from Magnetotactic Bacteria. Contrast Media Mol. Imaging 2016, 11, 139-145.

(10) Martel, S. Targeting active cancer cells with smart bullets. Ther. Delivery 2017, 8, 301-312.

(11) Felfoul, O.; Mohammadi, M.; Taherkhani, S.; de Lanauze, D.; Zhong Xu, Y.; Loghin, D.; Essa, S.; Jancik, S.; Houle, D.; Lafleur, M.; et al. Magneto-aerotactic bacteria deliver drug-containing nanoliposomes to tumour hypoxic regions. Nat. Nanotechnol. 2016, 11, 941-947.

(12) Mathuriya, A. S. Magnetotactic bacteria: nanodrivers of the future. Crit. Rev. Biotechnol. 2016, 36, 788-802.

(13) Gandia, D.; Gandarias, L.; Rodrigo, I.; Robles-García, J.; Das, R.; Garaio, E.; García, J. A.; Phan, M.; Srikanth, H.; Orue, I. Unlocking the Potential of Magnetotactic Bacteria as Magnetic Hyperthermia Agents. Small 2019, 15, 1902626.

(14) Larumbe, S.; Gomez-Polo, C.; Pérez-Landazábal, J. I.; GarcíaPrieto, A.; Alonso, J.; Fdez-Gubieda, M. L.; Cordero, D.; Gómez, J. Ni Doped $\mathrm{Fe}_{3} \mathrm{O}_{4}$ Magnetic Nanoparticles. J. Nanosci. Nanotechnol. 2012, $12,2652-2660$.

(15) Staniland, S.; Williams, W.; Telling, N.; Van Der Laan, G.; Harrison, A.; Ward, B. Controlled cobalt doping of magnetosomes in vivo. Nat. Nanotechnol. 2008, 3, 158-162.

(16) Tanaka, M.; Brown, R.; Hondow, N.; Arakaki, A.; Matsunaga, T.; Staniland, S. Highest levels of $\mathrm{Cu}, \mathrm{Mn}$ and $\mathrm{Co}$ doped into nanomagnetic magnetosomes through optimized biomineralisation. J. Mater. Chem. 2012, 22, 11919-11921.

(17) Li, J.; Menguy, N.; Arrio, M.-A.; Sainctavit, P.; Juhin, A.; Wang, Y.; Chen, H.; Bunau, O.; Otero, E.; Ohresser, P.; et al. Controlled cobalt doping in the spinel structure of magnetosome magnetite: new evidences from element- and site-specific X-ray magnetic circular dichroism analyses. J. R. Soc., Interface 2016, 13, No. 20160355.

(18) Marcano, L.; Muñoz, D.; Martín-Rodríguez, R.; Orue, I.; Alonso, J.; García-Prieto, A.; Serrano, A.; Valencia, S.; Abrudan, R.; Fernández Barquín, L.; et al. Magnetic Study of Co-Doped Magnetosome Chains. J. Phys. Chem. C 2018, 122, 7541-7550.

(19) Alphandèry, E.; Carvallo, C.; Menguy, N.; Chebbi, I. Chains of Cobalt Doped Magnetosomes Extracted from AMB-1 Magnetotactic Bacteria for Application in Alternative Magnetic Field Cancer Therapy. J. Phys. Chem. C 2011, 115, 11920-11924.

(20) Pérez-Gonzalez, T.; Prozorov, T.; Yebra-Rodríguez, A.; Bazylinski, D.; Jimenez-López, C. Mn incorporation in magnetosomes: New possibilities for the nanotechnological applications of biomagnetite. Macla 2010, 13, 171.

(21) Prozorov, T.; Perez-Gonzalez, T.; Valverde-Tercedor, C.; Jimenez-Lopez, C.; Yebra-Rodriguez, A.; Körnig, A.; Faivre, D.; Mallapragada, S. K.; Howse, P. A.; Bazylinski, D. A.; et al. Manganese incorporation into the magnetosome magnetite: magnetic signature of doping. Eur. J. Mineral. 2014, 26, 457-471.

(22) Muñoz, D.; Marcano, L.; Martín-Rodríguez, R.; Simonelli, L.; Serrano, A.; García-Prieto, A.; Fdez-Gubieda, M. L.; Muela, A. Magnetosomes could be protective shields against metal stress in magnetotactic bacteria. Sci. Rep. 2020, 10, No. 11430.

(23) Goldman, A. Modern Ferrite Technology; Springer Science \& Business Media, 2006.

(24) Vamvakidis, K.; Katsikini, M.; Vourlias, G.; Angelakeris, M.; Paloura, E. C.; Dendrinou-Samara, C. Composition and hydrophilicity control of $\mathrm{Mn}$-doped ferrite $\left(\mathrm{Mn}_{x} \mathrm{Fe}_{3-x} \mathrm{O}_{4}\right)$ nanoparticles induced by polyol differentiation. Dalton Trans. 2015, 44, 5396-5406.

(25) Lasheras, X.; Insausti, M.; de la Fuente, J. M.; Gil de Muro, I.; Castellanos-Rubio, I.; Marcano, L.; Fernández-Gubieda, M. L.; Serrano, A.; Martín-Rodríguez, R.; Garaio, E.; et al. Mn-Doping level dependence on the magnetic response of $\mathrm{Mn}_{\mathrm{x}} \mathrm{Fe}_{3-\mathrm{x}} \mathrm{O}_{4}$ ferrite nanoparticles. Dalton Trans. 2019, 48, 11480-11491.

(26) Keim, C. N.; Lins, U.; Farina, M. Manganese in biogenic magnetite crystals from magnetotactic bacteria. FEMS Microbiol. Lett. 2009, 292, 250-253.

(27) Heyen, U.; Schüler, D. Growth and magnetosome formation by microaerophilic Magnetospirillum strains in an oxygen-controlled fermentor. Appl. Microbiol. Biotechnol. 2003, 61, 536-544.

(28) Grunberg, K.; Wawer, C.; Tebo, B. M.; Schuler, D. A Large Gene Cluster Encoding Several Magnetosome Proteins Is Conserved in Different Species of Magnetotactic Bacteria. Appl. Environ. Microbiol. 2001, 67, 4573-4582.

(29) Schneider, C. A.; Rasband, W. S.; Eliceiri, K. W. NIH Image to ImageJ: 25 years of image analysis. Nat. Methods 2012, 9, 671.

(30) Castro, G. R. Optical design of the general-purpose Spanish Xray beamline for absorption and diffraction. J. Synchrotron Radiat. 1998, 5, 657-660.

(31) Ravel, B.; Newville, M. ATHENA, ARTEMIS, HEPHAESTUS: data analysis for X-ray absorption spectroscopy using IFEFFIT. $J$. Synchrotron Radiat. 2005, 12, 537-541.

(32) Grabis, J.; Nefedov, A.; Zabel, H. Diffractometer for soft x-ray resonant magnetic scattering. Rev. Sci. Instrum. 2003, 74, 4048-4051.

(33) Abrudan, R.; Brussing, F.; Salikhov, R.; Meermann, J.; Radu, I.; Ryll, H.; Radu, F.; Zabel, H. ALICE - An advanced reflectometer for static and dynamic experiments in magnetism at synchrotron radiation facilities. Rev. Sci. Instrum. 2015, 86, 063902.

(34) Å Tichauer, L.; Mirone, A.; Turchini, S.; Prosperi, T.; Zennaro, S.; Zema, N.; Lama, F.; Pontin, R.; ÅimÅ!a, Z.; Tailhades, P.; et al. Xray absorption spectroscopy and magnetic circular dichroism of the Mn-ferrite nanocrystalline thin films. J. Appl. Phys. 2001, 90, 25112516.

(35) Stavitski, E.; de Groot, F. M. The CTM4XAS program for EELS and XAS spectral shape analysis of transition metal L edges. Micron 2010, 41, 687-694.

(36) Bender, P.; Marcano, L.; Orue, I.; Alba Venero, D.; Honecker, D.; Fernández Barquín, L.; Muela, A.; Fdez-Gubieda, M. L. Probing the stability and magnetic properties of magnetosome chains in freeze-dried magnetotactic bacteria. Nanoscale Adv. 2020, 2, 11151121.

(37) Arato, B. Crystal-size and shape distributions of magnetite from uncultured magnetotactic bacteria as a potential biomarker. Am. Mineral. 2005, 90, 1233-1240.

(38) Pósfai, M.; Cziner, K.; Máton, E.; Márton, P.; Buseck, P. R.; Frankel, R. R.; Bazylinski, D. A. Crystal-size distributions and possible biogenic origin of Fe sulfides. Eur. J. Mineral. 2001, 13, 691-703.

(39) Fdez-Gubieda, M. L.; Muela, A.; Alonso, J.; Garcia-Prieto, A.; Olivi, L.; Fernandez-Pacheco, R.; Barandiaran, J. M. Magnetite Biomineralization in Magnetospirillum gryphiswaldense: Time-Resolved Magnetic and Structural Studies. ACS Nano 2013, 7, 3297-3305.

(40) Marcano, L.; García-Prieto, A.; Muñoz, D.; Fernández Barquín, L.; Orue, I.; Alonso, J.; Muela, A.; Fdez-Gubieda, M. Influence of the bacterial growth phase on the magnetic properties of magnetosomes synthesized by Magnetospirillum gryphiswaldense. Biochim. Biophys. Acta, Gen. Subj. 2017, 1861, 1507-1514.

(41) Mazarío, E.; Mayoral, A.; Salas, E.; Menéndez, N.; Herrasti, P.; Sanchez-Marcos, J. Synthesis and characterization of manganese ferrite nanoparticles obtained by electrochemical/chemical method. Mater. Des. 2016, 111, 646-650.

(42) Fdez-Gubieda, M. L.; García-Prieto, A.; Alonso, J.; Meneghini, C. X-Ray Absorption Fine Structure Spectroscopy in Fe Oxides and Oxyhydroxides. In Iron Oxides: From Nature to Applications; Faivre, D., Ed.; Wiley-VCH, 2016; pp 397-422.

(43) Farges, F. $A b$ initio and experimental pre-edge investigations of the Mn K -edge XANES in oxide-type materials. Phys. Rev. B 2005, 71, No. 155109.

(44) Bargar, J.; Tebo, B.; Villinski, J. In situ characterization of $\mathrm{Mn}$ (II) oxidation by spores of the marine Bacillus sp. strain SG-1. Geochim. Cosmochim. Acta 2000, 64, 2775-2778. 
(45) McKeown, D. A.; Post, J. E. Characterization of manganese oxide mineralogy in rock varnish and dendrites using X-ray absorption spectroscopy. Am. Mineral. 2001, 86, 701-713.

(46) McKeown, D. A.; Kot, W. K.; Gan, H.; Pegg, I. L. X-ray absorption studies of manganese valence and local environment in borosilicate waste glasses. J. Non-Cryst. Solids 2003, 328, 71-89.

(47) Wilke, M.; Farges, F.; Petit, P.-E.; E. Brown, G., Jr.; Martin, F. Oxidation state and coordination of Fe in minerals: An Fe K-XANES spectroscopy study. Am. Mineral. 2001, 86, 714-730.

(48) Westre, T. E.; Kennepohl, P.; DeWitt, J. G.; Hedman, B.; Hodgson, K. O.; Solomon, E. I. Multiplet Analysis of Fe K-Edge 1s $\rightarrow$ 3d Pre-Edge Features of Iron Complexes. J. Am. Chem. Soc. 1997, 119, $6297-6314$.

(49) van der Laan, G.; Figueroa, A. I. X-ray magnetic circular dichroism-A versatile tool to study magnetism. Coord. Chem. Rev. 2014, 277-278, 95-129.

(50) Pattrick, R. A. D.; Van Der Laan, G.; Henderson, C. M. B.; Kuiper, P.; Dudzik, E.; Vaughan, D. J. Cation site occupancy in spinel ferrites studied by X-ray magnetic circular dichroism: developing a method for mineralogists. Eur. J. Mineral. 2002, 14, 1095-1102.

(51) O'Handley, R. Modern Magnetic Materials: Principles and Applications; Wiley, 1999.

(52) Carta, D.; Casula, M. F.; Mountjoy, G.; Corrias, A. Formation and cation distribution in supported manganese ferrite nanoparticles: an X-ray absorption study. Phys. Chem. Chem. Phys. 2008, 10, 31083117.

(53) Liang, X.; Zhong, Y.; Zhu, S.; He, H.; Yuan, P.; Zhu, J.; Jiang, Z. The valence and site occupancy of substituting metals in magnetite spinel structure $\mathrm{Fe}_{3-x} \mathrm{M}_{x} \mathrm{O}_{4}(\mathrm{M}=\mathrm{Cr}, \mathrm{Mn}, \mathrm{Co}$ and $\mathrm{Ni})$ and their influence on thermal stability: An XANES and TG-DSC investigation. Solid State Sci. 2013, 15, 115-122.

(54) Walz, F. The Verwey transition - a topical review. J. Phys. Condens. Matter. 2002, 14, R285-R340.

(55) Prozorov, R.; Prozorov, T.; Mallapragada, S. K.; Narasimhan, B.; Williams, T. J.; Bazylinski, D. A. Magnetic irreversibility and the Verwey transition in nanocrystalline bacterial magnetite. Phys. Rev. B 2007, 76, No. 054406.

(56) Fernandez, E.; Tu, K.-H.; Ho, P.; Ross, C. A. Thermal stability of L10-FePt nanodots patterned by self-assembled block copolymer lithography. Nanotechnology 2018, 29, No. 465301.

(57) Fischer, H.; Mastrogiacomo, G.; Loffler, J. F.; Warthmann, R. J.; Weidler, P. G.; Gehring, A. U. Ferromagnetic resonance and magnetic characteristics of intact magnetosome chains in Magnetospirillum gryphiswaldense. Earth Planet. Sci. Lett. 2008, 270, 200-208.

(58) Kronmüller, H.; Walz, F. Magnetic after-effects in $\mathrm{Fe}_{3} \mathrm{O}_{4}$ and vacancy-doped magnetite. Philos. Mag. B 1980, 42, 433-452.

(59) Muxworthy, A. R.; McClelland, E. Review of the lowtemperature magnetic properties of magnetite from a rock magnetic perspective. Geophys. J. Int. 2000, 140, 101-114.

(60) Stoner, E.; Wohlfarth, E. A mechanism of magnetic hysteresis in heterogenious alloys. Philos. Trans. Royal Soc. A 1948, 240, 599-642.

(61) Orue, I.; Marcano, L.; Bender, P.; García-Prieto, A.; Valencia, S.; Mawass, M. A.; Gil Carton, D.; Alba Venero, D.; Honecker, D.; García-Arribas, A.; et al. Configuration of the magnetosome chain: a natural magnetic nanoarchitecture. Nanoscale 2018, 10, 7407-7419.

(62) Huizar-Félix, A. M.; Muñoz, D.; Orue, I.; Magen, C.; Ibarra, A.; Barandiaran, J. M.; Muela, A.; Fdez-Gubieda, M. L. Assemblies of magnetite nanoparticles extracted from magnetotactic bacteria: A magnetic study. Appl. Phys. Lett. 2016, 108, No. 063109.

(63) Charilaou, M.; Kind, J.; García-Rubio, I.; Schüler, D.; Gehring, A. U. Magnetic anisotropy of non-interacting collinear nanocrystalchains. Appl. Phys. Lett. 2014, 104, No. 112406.

(64) Charilaou, M.; Winklhofer, M.; Gehring, A. U. Simulation of ferromagnetic resonance spectra of linear chains of magnetite nanocrystals. J. Appl. Phys. 2011, 109, No. 093903.

(65) Gehring, A. U.; Fischer, H.; Charilaou, M.; García-Rubio, I. Magnetic anisotropy and Verwey transition of magnetosome chains in Magnetospirillum gryphiswaldense: Magnetic anisotropy and Verwey transition. Geophys. J. Int. 2011, 187, 1215-1221.
(66) Peddis, D.; Muscas, G.; Mathieu, R.; Kumar, P. A.; Varvaro, G.; Singh, G.; Orue, I.; Gil-Carton, D.; Marcano, L.; Muela, A.; et al. Studying nanoparticles' 3D shape by aspect maps: Determination of the morphology of bacterial magnetic nanoparticles. Faraday Discuss. 2016, 191, 177-188.

(67) Gandia, D.; Gandarias, L.; Marcano, L.; Orue, In.; Gil-Cartón, D.; Alonso, J.; García-Arribas, A.; Muela, A.; Fdez-Gubieda, M. Elucidating the role of shape anisotropy in faceted magnetic nanoparticles using biogenic magnetosomes as a model. Nanoscale 2020, 12, 16081-16090.

(68) Geoghegan, L. J.; Coffey, W. T.; Mulligan, B. Differential Recurrence Relations for Non-Axially Symmetric Rotational FokkerPlanck Equations. Adv. Chem. Phys. 1997, 100, 475-641.

(69) Carrey, J.; Mehdaoui, B.; Respaud, M. Simple models for dynamic hysteresis loop calculations of magnetic single-domain nanoparticles: Application to magnetic hyperthermia optimization. J. Appl. Phys. 2011, 109, No. 083921.

(70) Chikazumi, S. Physics of Ferromagnetism 2e; OUP Oxford, 2009.

(71) Penoyer, R. F.; Shafer, M. W. On the Magnetic Anisotropy in Manganese-Iron Spinels. J. Appl. Phys. 1959, 30, S315-S316.

(72) Alphandéry, E.; Ding, Y.; Ngo, A. T.; Wang, Z. L.; Wu, L. F.; Pileni, M. P. Assemblies of Aligned Magnetotactic Bacteria and Extracted Magnetosomes: What Is the Main Factor Responsible for the Magnetic Anisotropy. ACS Nano 2009, 3, 1539-1547. 